collection/ sharing of data to sustain delivery of quality care is developed. Identify Key Champions within each ICU who will take responsibility locally for implementation. Supported by the Project LCP ICU Facilitator to, deliver robust education and training and facilitate and monitor the implementation process. Key Champions meet monthly with the Project LCP ICU Facilitator to discuss aspects of the project.

Results Organisational information from each ICU will be collected pre and post implementation. Data from completed LCP ICU's in each unit will be analysed descriptively to provide a comparative benchmark of care delivered across the CMCCN. Reflective diaries kept by Key Champions and the LCP ICU Facilitator throughout the project will be analysed thematically to derive an understanding of the process of implementation, facilitating factors, barriers and challenges.

Conclusion Findings from all data collected will be presented, discussing implications for wider implementation of the LCP ICU V12.

\section{P 08 IMPLEMENTING THE LIVERPOOL CARE PATHWAY FOR THE DYING PATIENT IN INTENSIVE CARE VERSION 12 (LCP ICU V12) ACROSS A REGIONAL CRITICAL CARE NETWORK}

D. Parsons ${ }^{1,2}$, M. Gambles', L. Chapman',2 ${ }^{1}$ Marie Curie Palliative Care Institute Liverpool, University of Liverpool; ${ }^{2}$ Directorate of Specialist Palliative Care, 1st Floor Linda McCartney Centre, Royal Liverpool and Broadgreen University Hospitals NHS Trust, Prescot Street, Liverpool Merseyside L7 8XP

10.1136/bmjspcare-2012-000264.15

Introduction A pilot project is underway in 11 intensive care units within the Cheshire and Merseyside Critical Care Network (CMCCN) to implement the Liverpool Care Pathway for the Dying Patient in Intensive Care Version 12 (LCP ICU V12). Funded by Merseyside and Cheshire Cancer Network, this project will provide support to ICU's to implement and sustain the LCP ICU V12

Aims and Methods Implement LCP ICU V12 into 11 sites within the CMCCN and ensure. Processes and policies are in place to support care in the last hours or days of life. Staff are appropriately trained to deliver care. A mechanism for ongoing 